\title{
Review on: Data Aggregation and Dynamic Routing in Wireless Sensor Network
}

\author{
Yuoraj Somkuwar \\ Dept. of Computer Engineering \\ Smt. Radhikatai Pandav College of Engineering \\ Nagpur, India
}

\author{
S.D.Kamble \\ Professor, Dept. of Computer Technology \\ Yeshwantrao Chavan College of Engineering \\ Nagpur, India
}

\begin{abstract}
A wireless sensor network (WSN) has important applications such as monitoring temperature, humidity, level, speed, remote environmental monitoring and target tracking. Main critical issue in sensor network is maximization of network lifetime. All sensor nodes are required large amount of energy to send and receive data; also it depends upon distance between two nodes. Therefore with the help of Data aggregation technique we reduce the size of data. In wireless sensor network's (WSN's) nodes need less power for processing as compared to send data. Second technique is to reduce amount of energy to send data by using shortest path that can be achieve with the help of dynamic routing. Our proposed technique is attribute aware data aggregation. Attributes means the identifier of sampled data by different sensors node such as humidity sensors, temperature sensors etc., should be gathered together. Attribute-aware Data Aggregation mechanism which can collect packets with same attribute as much as possible and hence improve the efficiency of data aggregation. This aim can't be achieved by static routing technique of data aggregation mechanisms since they construct routes before transmitting the sampled data and thus cannot dynamically forward packets in response to the variation of packets at intermediate. Therefor we use potential-based dynamic routing scheme to transfer data into network.
\end{abstract}

General terms: Routing, Aggregation, Static Routing.

Keyword: Wireless Sensor Networks, Dynamic routing, Data Aggregation, Attribute aware, Potential based.

\section{INTRODUCTION}

Wireless sensor is collection of hundreds or thousands of sensor node, thus it has various applications such as habitat and environment monitoring, fire detection, intrusion detection, biological hazard detection and intrusion detection [2]. Normally sensor nodes are low-powered sensing devices with, less computational capacity, limited memory and communication resources [1]. Wireless sensor networks have tiny devices hence resources are very limited. Large amount of energy required for transmitting data and receiving data from node to node, it also depends upon distance between nodes. Main issue in WSN is to increase the lifetime of network that can be improved with the help of data aggregation mechanism. Data aggregation mechanism is the process of collection of data coming from different neighboring sensor node and removing redundancy from data after that send those data to base station with appropriate routing mechanism due to which improve the overall performance of network and maximize lifetime of network.

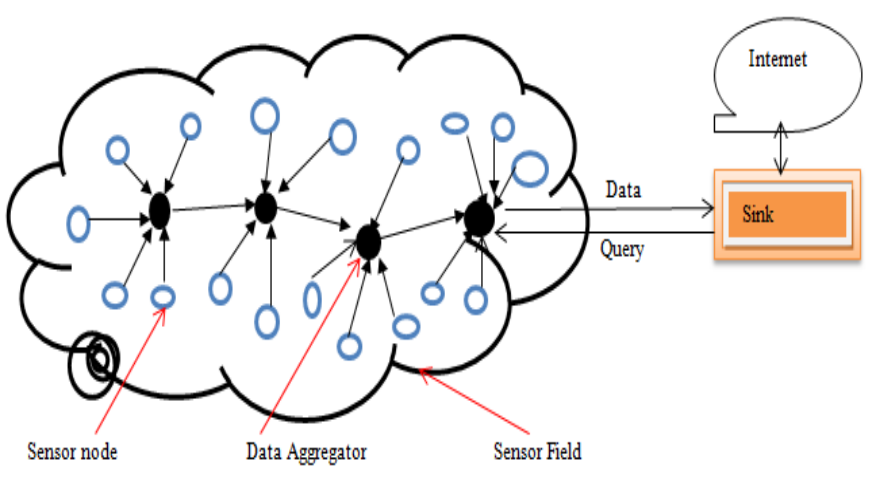

Fig 1.Overview of Data Aggregation WSN

In wireless sensor network advantage of data aggregation increases when intermediate sensor nodes perform data aggregation mechanism and data are forwarded to the sink. Data aggregation operation perform continuously due to which improves the bandwidth and energy utilization, it may negatively effect of other performance metrics such as delay, fault-tolerance and accuracy, etc. [2]. Main Goal of data aggregation mechanism to sorts data and make packets more spatially as well as temporally convergent to reduce Average Number of Transaction (ANT). For example, in [3], if two or more kind of sensors, such as pressure sensors, traffic sensor are working in a same area. The packets generated by the sensor nodes are transmitted to base station. Attribute is defined as identification of packet [4]. In this paper we use Attribute Aware Data Aggregation i.e. it identified the type of packets. If packet having same attribute then apply Aggregation mechanism on that node. Although the timing scheme proposed in [3] ensures that all the packet of same Attribute meet each other at same time for Aggregation. Static routing is define as per define path between source node to destination node and that is fixed due to which arise a problem such as packet loss, traffic conjunction, packet delay and so on. Dynamic routing is defined as path can be decided at the run time of packet form source to destination [20]. To design the dynamic routing, first we know that the routing metric that can be determined on node depth, is determined how many number of hop is require reaching sink node, is considered to ensure packets reach the sink at last. Besides, the metric must be relevant with the packet attribute. Enlightened by the concept of pheromone, which will be drop along the path where the ants pass and evaporate with time, in ant colony [17], we draw an analogy between the pheromone and the packet attribute. A packet will drop attribute-dependent pheromone when packet passing a node to attract towards packets with same attribute node .In this paper remaining wok is II Related work. III proposed technique. IV Conclusion. 


\section{RELATED WORK}

\subsection{Data Aggregation}

Wireless sensor network are very challenging research area for design Energy efficient routing protocols. Sensor nodes are tiny device due which resources is very less hence traditional routing protocols are not suitable for WSN[20]. One of the most suitable methods is data aggregation in WSN. Data aggregation is gathering data from neighboring node, removal of redundant data from that node hence reducing the number of packets transmitted over the network because processing data for aggregation required less power as compare sending multiple packets having similar data [5]. There is different type of algorithms discus in literature for performing aggregation. These mechanisms are of three types such as in network data aggregation, hybrid data aggregations and global data aggregation scheme [6] Proposed an in network data aggregation scheme for location aware routing in wireless sensor networks. In order to reduce the size of transmitting packets over the networks, they utilize group by queries. The weakness of this lies in construction mechanism of routing tree which is random. For information retrieval, base station broadcast a message and next node to be selected is the one who broadcasts the base station message first. In chain based data aggregation mechanism [7] sensor nodes send their data to the closest neighbors. Aggregation activities are done at the cluster heads. Ordinary nodes sense data and send to their cluster head which is forwarded to a base station or other cluster head but it apply aggregation process before send data from one cluster head to another. However, if distance between cluster head and ordinary node is large then energy required to transmit data is large. In [11] direct diffusion was presented which is focused on reducing the size of data retrieved from different sources by eliminating redundancy and reducing the number of packets transmitted over the network. But the problem with this approach is the identification of alternative optimal path when the current path is lost. But the problem with this approach is the identification of alternative optimal path when the current path is lost Gradient based routing [8] is next version of direct diffusion in which traffic is distributing evenly due to this the network lifetime is maximized. In this mechanism, sensor nodes always know that depth of node form based station also called the height of nodes. The packets are forwarded over that link having largest gradient values. The gradient is obtained by subtracting a node's height from that of its neighbor nodes. A new deterministic data aggregation method for wireless sensor networks was presented in [9]. This technique uses a deterministic sampling approach in comparison to random sampling. The size of resulting sample is very small and requires less transmission power which results in prolonging network lifetime. Data compression is also used as an aggregation method in wireless sensor network [10] in this method use piecewise approximation algorithm which compresses data. Due to the reduced size of packet, hence the network lifetime is increased. The disadvantage of this algorithm is the loss of data as well as it is useful to single dimensional time series. Data aggregation in tree based networks is performed at the intermediate nodes and is suitable for applications where in network data aggregation is required. In energy aware data aggregation tree algorithm [11], a control message is periodically broadcasted by the base station. The timer is started by each node on receiving this message and is refreshed if a new control message arrived during the time count down time. However, most of these algorithms were developed for special application and not generalized there always need for efficient energy aware and robust data aggregation algorithm.

\subsection{Related work on Routing in Wireless senor Network Location based protocol}

Location base protocol are design for node access by their address, location information of sensor node is required for sensor network by routing protocol and to calculate distance between source node and destination node so that it easy to find out the path between node.

Table No.1 summary of location based protocol

\begin{tabular}{|c|c|c|c|c|}
\hline $\begin{array}{l}\text { Sr. } \\
\text { no }\end{array}$ & protocol & Description(location based ) & Advantages & Limitation \\
\hline 1 & $\begin{array}{c}\text { Geographic } \\
\text { Random } \\
\text { Forwarding }(\mathrm{GeRaF})\end{array}$ & $\begin{array}{l}\text { Geographic routing is a routing where } \\
\text { sensor node is acting as relay which priori } \\
\text { unknown to a sender. There is no } \\
\text { assurance that massages reach to the exact } \\
\text { destination. A sender will always forward } \\
\text { the message toward its base station Best- } \\
\text { effort forwarding RTS/ a CTS message } \\
\text { mechanism are employed [12]. }\end{array}$ & $\begin{array}{l}\text { Reliability increases } \\
\text { due to Back off time. }\end{array}$ & $\begin{array}{l}\text { User involvement } \\
\text { Is necessary in each } \\
\text { stage, more time } \\
\text { gives efficient output }\end{array}$ \\
\hline 2 & $\begin{array}{l}\text { Minimum Energy } \\
\text { Communication } \\
\text { Network(MECN) }\end{array}$ & $\begin{array}{l}\text { Achieving less energy for randomly } \\
\text { deployed ad hoc networks and calculate } \\
\text { an optimal spanning tree which is rooted } \\
\text { at the sink. This is called minimum power } \\
\text { topology. It contains only the minimum } \\
\text { power paths from each sensor to the sink } \\
\text { [13]. }\end{array}$ & $\begin{array}{l}\text { Fault tolerant } \\
\text { Optimal spanning } \\
\text { Self-configuring }\end{array}$ & $\begin{array}{l}\text { Fault tolerant is } \\
\text { depend upon the } \\
\text { specific application }\end{array}$ \\
\hline 3 & $\begin{array}{l}\text { Small Minimum- } \\
\text { Energy } \\
\text { Communication } \\
\text { Network (SMECN) }\end{array}$ & $\begin{array}{l}\text { A routing protocol is improved version of } \\
\text { MECN.Here; a minimal graph is } \\
\text { characterized with regard to the minimum } \\
\text { energy property. }\end{array}$ & $\begin{array}{l}\text { A minimum energy } \\
\text { efficient path between } \\
\text { nodes }\end{array}$ & $\begin{array}{l}\text { No. of broadcast } \\
\text { messages is large }\end{array}$ \\
\hline
\end{tabular}

\subsection{Data Centric Protocols}

In data-centric protocols, source sensors send their data to the sink. After this, intermediate sensors perform some kind of aggregation on the data which is originating from multiple source sensors and then send the aggregated data toward the sink 
Table No.2. Summary of data centric protocol

\begin{tabular}{|c|c|c|c|c|}
\hline sr.no & protocol & Description & Advantages & Limitation \\
\hline 1 & $\begin{array}{l}\text { SPIN: Sensor } \\
\text { Protocols for } \\
\text { Information } \\
\text { Negotiation }\end{array}$ & $\begin{array}{l}\text { One of the most dominant forms of routing in the wireless } \\
\text { sensor networks [14]. } \\
\text { SPIN1: SPIN-1 is improved version of SPIN. It is a source } \\
\text { initiated, data centric, data aggregation and source initiated } \\
\text { protocol. It establishes a connection based on three-way } \\
\text { handshake [R2]. } \\
\text { M-Spin: Here, total number of packet transmissions is less. } \\
\text { Therefore a significant amount of total energy can be saved. } \\
\text { Also, energy consumption is not only depends upon sensing } \\
\text { of the data but on processing the sensed data and transmitting } \\
\text { or receiving them to or from its neighbor nodes. }\end{array}$ & $\begin{array}{lr}\text { Energy } & \text { aware, } \\
\text { Resource } & \text { aware } \\
\text { and } & \text { resource } \\
\text { adaptive } & \end{array}$ & $\begin{array}{l}\text { Applies only to } \\
\text { lossless } \\
\text { Networks Not } \\
\text { applicable in } \\
\text { mobile links }\end{array}$ \\
\hline 2 & $\begin{array}{l}\text { Energy- } \\
\text { Aware Data- } \\
\text { Centric } \\
\text { Routing } \\
\text { (EAD): }\end{array}$ & $\begin{array}{l}\text { Within a wireless sensor network, it is based on a low cost } \\
\text { backbone which turns off the non-backbone nodes and save } \\
\text { energy. Thus, extending the network lifetime without } \\
\text { compromising the connectivity of the network [R1]. }\end{array}$ & $\begin{array}{l}\text { Power saving. } \\
\text { Lifetime } \\
\text { increased. }\end{array}$ & $\begin{array}{l}\text { Gateway handling } \\
\text { required }\end{array}$ \\
\hline 3 & $\begin{array}{l}\text { Rumor } \\
\text { Routing }\end{array}$ & $\begin{array}{l}\text { Here, a long-lived packets traverse through the network and } \\
\text { inform s each sensor. These packets are known as Agent. It } \\
\text { Encounters the events that it has learned during its network } \\
\text { traverse. These agents traverse through certain number of } \\
\text { hopes in a network and then Die [15]. }\end{array}$ & $\begin{array}{l}\text { Under a wide } \\
\text { range of } \\
\text { conditions, Rumor } \\
\text { Routing algorithm } \\
\text { delivers queries to } \\
\text { the events in large } \\
\text { networks with } \\
\text { energy } \\
\text { requirements lower } \\
\text { than the } \\
\text { alternatives. }\end{array}$ & $\begin{array}{l}\text { The algorithm is } \\
\text { likely to favor } \\
\text { older events which } \\
\text { are not suitable for } \\
\text { the dynamics } \\
\text { Network and } \\
\text { asynchronous } \\
\text { events }\end{array}$ \\
\hline
\end{tabular}

\subsection{Single- multipath based protocol:}

When data transfer between source node and the sink, there are two Types routing is applied one of them is single-path (Static Routing) routing and other is multipath routing (Dynamic Routing). In static routing, each source node sends its sensed data to the sink through the shortest path. But, in multipath routing, first each source node finds the $\mathrm{K}$ shortest paths to the sink and then distributes its load evenly among those paths.

Table 3summary of Single- multipath based protocol

\begin{tabular}{|c|c|c|c|c|}
\hline Sr.no & Protocol & Description & Advantages & Limitation \\
\hline 1 & Disjoint Paths & $\begin{array}{l}\text { Using this protocol we find a short number of } \\
\text { alternate paths which have no common sensor } \\
\text { between each other and with the primary path. For } \\
\text { sensor network where disjoint path routing used, the } \\
\text { primary paths are best than the alternate paths as } \\
\text { they have longer latency and less efficiency. The } \\
\text { disjoint makes those alternate paths do not depend } \\
\text { upon of the primary path [18]. }\end{array}$ & $\begin{array}{l}\text { If a fault occurs on the } \\
\text { primary path, then it uses } \\
\text { alternate paths which } \\
\text { neighbors can provide more } \\
\text { quality data is determine by } \\
\text { the sink. These are } \\
\text { characterized by the lowest } \\
\text { loss or lowest delay after } \\
\text { the network flooded with } \\
\text { some low-rate samples. }\end{array}$ & $\begin{array}{l}\text { More chances of } \\
\text { node failures., less } \\
\text { energy \& energy } \\
\text { efficient }\end{array}$ \\
\hline 2 & Braided Paths & $\begin{array}{l}\text { To construct the braided multipath, first find out } \\
\text { primary path. After this, for every node (or sensor) } \\
\text { on the primary path, the best path from a source } \\
\text { sensor node to the sink node excluding that node is } \\
\text { computed. Those best alternate paths which are not } \\
\text { necessarily disjoint from the primary path, called } \\
\text { idealized braided multipath. }\end{array}$ & $\begin{array}{l}\text { Links of each of the } \\
\text { alternate paths is based on } \\
\text { to the primary path. Thus, } \\
\text { energy consumption on the } \\
\text { primary and alternate paths } \\
\text { seems to be comparable as } \\
\text { opposed to the scenario of } \\
\text { mutually ternate and } \\
\text { primary paths. }\end{array}$ & $\begin{array}{c}\text { Find multiple path } \\
\text { with less loss of } \\
\text { each path to the } \\
\text { other }\end{array}$ \\
\hline
\end{tabular}




\section{PROPOSED METHODOLOGY}

AS, shown in Figure 2, all sensor nodes placed into network for collect the data (temperature, pressure, humidity) according to query send by the base station. Base Station Broadcast the query into the network, then those sensor node are interest on that query send the sense result to its neighbor and neighbor node send data to its nearest neighbor in this way basic communication can be done in between the sensor node. Flowchart show that the typical representation of the flow of the proposed system. In first step Base station send the query into the network. Source node sense data and send response to its neighbor, we placed label on the packet called as header, when packet having same data then header on the two packet (existing packet, new received packet) having same value. In third step if both packet having form same application then apply data aggregation process on that intermediate node having no of packet with same header. If packet having different application then simply transmit to its neighbor node at one time it reach to the position having node as same packet ,then apply the data aggregation process on that all packet and remove the redundancy form data . In this process the data size is reduce hence required energy to transmitted data from source to destination is less because of data size in this way we can save the energy with the help of data aggregation process. After apply data aggregation method then reduce the energy with the help of dynamic routing. In this process network select the shortest path between the source and destination node. At the last step data reach to the base station.

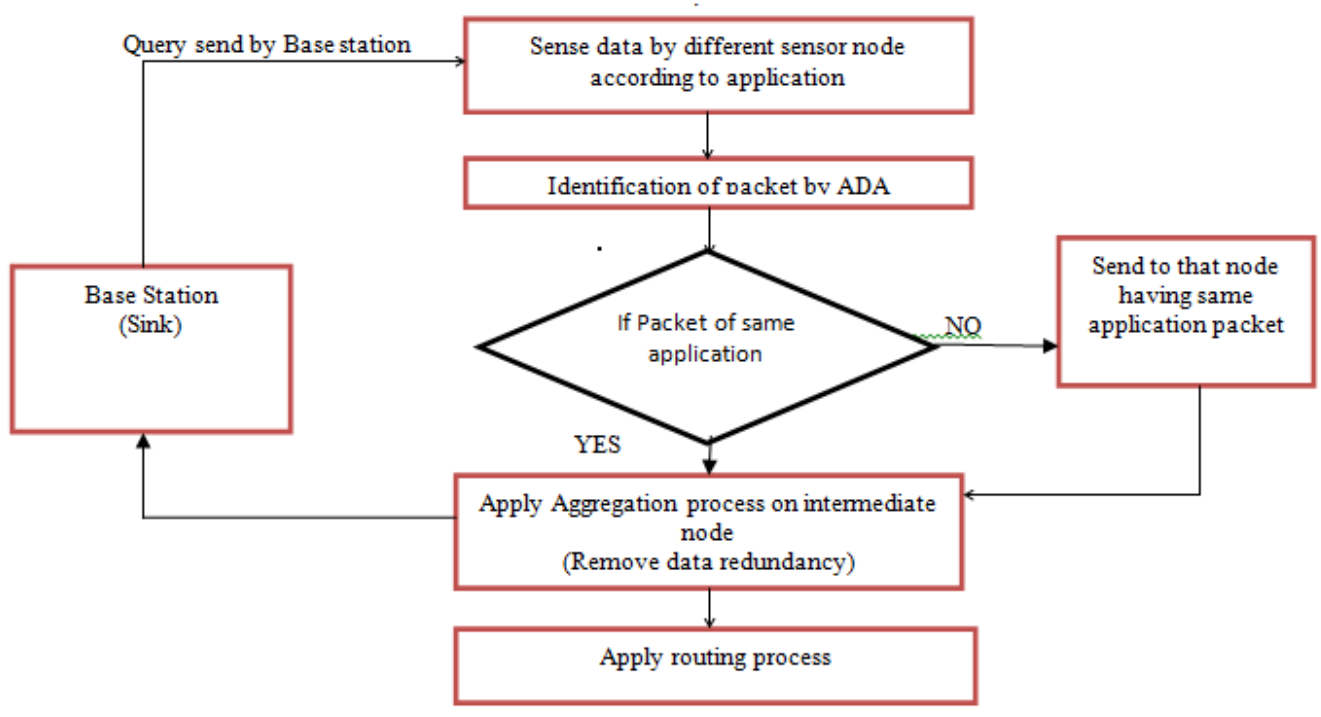

Fig 2. Aggregation process

\section{CONCLUSION}

Unlike other networks, WSNs are designed for specific applications. Applications, but are not limited to, industrial machine monitoring, environmental monitoring, industrial machine monitoring, monitoring temperature, humidity, level, military target tracking, speed and surveillance systems. Each application differs in requirements and features. To support this diversity of applications that development of new communication protocols, services are needed and algorithms, designs,. We have surveyed in this paper issues on of make system with more energy efficient categories: (1) Data Aggregation, (2) Routing protocol. We have investigated the impact of data aggregation in establishing routing paths towards the sink for the energy minimization problem. For Attribute aware routing will be used in our proposed system. We have summarized and compared different existing algorithms, protocols, and services. Moreover, we have highlighted possible research and improvements in each area. There are still many issues to be resolved around WSN applications such as communication architectures, security, and management and lifetime of network. By solving these issues, we can close the gap between technology and application.

\section{REFERENCES}

[1] I.F. Akyildiz, W. Su, Y. Sankarasubramaniam, E. Cayirci, 2002, "A survey on sensor networks", IEEE Commun. Mag. 40 (8),p: 102-114.

[2] K. Akkaya, M. Demirbas, R.S. Aygun, 2008, "The Impact of Data Aggregation on the Performance of Wireless Sensor Networks", Wiley Wireless Commun. Mobile Comput. (WCMC) J. 8, p: 171-193.

[3] I. Solis and K. Obraczka, 2004, "In-Network Aggregation Trade-offs for Data Collection in Wireless Sensor Networks," in University of California Santa Cruz Technical Report,

[4] Jiao Zhang, Fengyuan Ren, Tao He , Chuang Lin, 2010, "Attribute-aware Data Aggregation Using DynamicRouting in Wireless Sensor Networks", Tsinghua National Laboratory for Information Science and Technology, Beijing 100084, ChinaDept. of Computer Science and Technology, Tsinghua University, Beijing, 100084, IEEE

[5] Kiran Maraiya, K.K., Nitin Gupta, 2011, "Wireless Sensor Network: A Review on Data Aggregation", IJSCR. 
[6] Jonathan Beaver, M.A.S., Alexandros Labrinidis , Ros Labrinidis , Panos K. Chrysanthis, 2003, "Locationaware routing for data aggregation in sensor Networks". Geosensor Networks.

[7] Ramesh Rajagopalan , P.K.V., 2006 "Data aggregation techniques in sensor networks: A survey", Comm. Surveys \& Tutorials, IEEE, 8: p. 48-63.

[8] Yoo, H.K.N.U., Daegu, South Korea, M.K. Shim, DongKyun Kyun; Kyu Hyung Kim, 2010, "GLOBAL: A Gradient-based routing protocol for Load-balancing in large-scale wireless sensor networks with multiple sinks" .IEEE Symposium on Computers and Communications (ISCC), :p. 556-562

[9] Hüseyin Akcan , H.B., 2007, “A New Deterministic Data Aggregation Method For Wireless Sensor Networks Signal Processing”. 87(12): p. 2965-2977.

[10] Xiao-fei, Y.A.S., Xiao-bei, Wu;Jin-an, Huang,2009, "TAGPP: A Tiny Aggregation Algorithm with Preprocessing in Local Cluster. International Conference on Networks Security, Wireless Communications and Trusted Computing",. 2: p. 390-393

[11] Zhao, S.Y., Fengqi Qi, Zhao, Bao-Hua Hua,2007, "An Energy Efficient Directed Diffusion Routing Protocol" International Conference on Computational Intelligence and Security: p. 1067-1072

[12] M. Zorzi and R. R. Rao, Oct.-Dec. 2003, "Geographic random forwarding $(\mathrm{GeRaF})$ for ad hoc and sensor networks: Mutlihop performance", IEEE Transactions on mobile Computing", vol. 2, no. 4, pp. 337-348.

[13] V. Rodoplu and T. H. Meng, "Minimum energy mobile wireless networks", IEEE Journal on Selected Areas in Communications, vol. 17, no. 8, Aug. 1999, pp. 13331344.
[14] W. R. Heinzelman, J. Kulik, and H. Balakrishnan, Aug.1999,"Adaptiveprotocols for information dissemination in wireless sensornetworks", ACM.

[15] David Braginsky, Deborah Estrin, 2002 "Rumor Routing Algorithm For Sensor Networks", WSNA'02, ACM.

[16] LA CA 90095-1596N. Sadagopan, B. Krishnamachari, and A. Helmy, 2003, "The ACQUIRE mechanism for efficient querying in sensor networks", Proceedings SNPA'03, Anchorage, AK, May, pp. 149-155.

[17] M. Dorigo, V. Maniezzo, and A. Colorni, 2002, "The Ant System: Optimization by a Colony of Cooperating Agents," IEEE.

[18] S. Lindsey, C. S. Raghavendra, and K. M. Sivalingam,Apr. 2001, "Data gathering in sensor networks using the energy delay metric", PDPS'01.

[19] M. Chu, H. Haussecker, and F. Zhao, Feb. 2002, "Scalable informationdriven sensor querying and routing for ad hoc heterogeneous sensor networks", vol. 16, no. 3, pp. 293-31, IJHPCA.

[20] Bappaditya Das1, Utpal Biswas, Debabrata Sarddar, 2013, "Electrostatic Field Based Reliable Routing In WirelessSensor Networks Using Vector Method", ISSN: 2231- 1963, IJAET

[21] K. Karthikeyan, M.Kavitha, 2013, "Comparative Analysis Of Data Centric Routing Protocols For Wireless Sensor Networks", Volume 3, Issue 1, IJSRP.

[22] Azzedine Boukerche, Xuzhen Cheng, Joseph Linus 2005, "A Performance Evaluation Of A Novel EnergyAware Data-Centric Routing Algorithm In Wireless Sensor Networks", Springer. 